\title{
Hiperplasia prostática e os principais sintomas do aparelho genito- urinário na população masculina de Coari - Amazonas, Brasil
}

\author{
Prostatic hyperplasia and the main genital urinary tract symptoms in the male \\ population of Coari - Amazonas, Brazil \\ Hiperplasia prostática y los principales síntomas del aparato genito-urinario en la \\ población masculina de Coari - Amazonas, Brasil
}

Lucas da Silva de Almeida ${ }^{1 *}$, Ricardo dos Santos Faria ${ }^{1}$, Priscilla Mendes Cordeiro ${ }^{1}$, Marcelo Brendew Sousa de Oliveira ${ }^{1}$, Rhuana Maria de Oliveira Pereira ${ }^{2}$, Vanessa de Souza Correia de Araujo $^{2}$, Yana Azevedo das Chagas ${ }^{3}$, Felipe Lopes de Souza ${ }^{4}$, Paula Andreza Viana Lima ${ }^{1}$, Jéssica Karoline Alves Portugal ${ }^{1}$.

\section{RESUMO}

Objetivo: Determinar a prevalência da Hiperplasia Prostática Benigna e os principais sintomas do aparelho genito-urinário em indivíduos que fazem uso do serviço público de saúde, residentes no município de Coari, Amazonas. Métodos: Trata-se de um estudo transversal, descritivo e observacional realizados com 106 homens a partir de 40 anos. Resultados: A prevalência de Hiperplasia Prostática Benigna na população masculina de Coari - Amazonas foi de 51,9\%. Homens com faixa etária de 61 a 70 anos e idade igual ou superior a 71 anos tiveram as maiores taxas de alteração do volume da próstata $\mathrm{O}$ escore da intensidade dos sintomas no trato urinário inferior encontrado na maioria dos indivíduos pesquisados foram leves e o grau de disfunção erétil foi de leve a moderado. Conclusão: A Hiperplasia Prostática Benigna foi prevalente na maioria dos homens acima de 40 anos que fizeram uso dos serviços públicos de saúde do município de Coari e a intensidade dos sintomas do aparelho genito-urinário foram de leve a moderado. Esses dados servem de alerta para os profissionais de saúde do município sobre a presença desse agravo e mostram a necessidade de intensificar ou continuar as atividades de saúde voltadas a este público.

Palavras-chave: Hiperplasia prostática, Saúde do homem, Sistema urinário.

\begin{abstract}
Objective: To determine the prevalence of Benign Prostatic Hyperplasia and the main symptoms of the genitourinary system in individuals who make use of the public health service, residents in the city of Coari, Amazonas. Methods: This is a cross-sectional, descriptive and observational study conducted with 106 men over 40 years of age. Results: The prevalence of Benign Prostatic Hyperplasia in the male population of Coari - Amazonas was $51.9 \%$. Men aged 61 to 70 years and aged 71 years or older had the highest rates of change in prostate volume The score of symptom intensity in the lower urinary tract found in most of the individuals surveyed were mild and the degree of dysfunction Erectile was mild to moderate. Conclusion: Benign prostatic hyperplasia was prevalent in most men older than 40 years who made use of public health services in the city of Coari and the intensity of genital urinary tract symptoms was mild to moderate. These data serve as a warning to health professionals in the municipality about the presence of this disease and show the need to intensify or continue health activities aimed at this public.
\end{abstract}

Keywords: Prostatic hyperplasia, Men's health, Urinary tract.

\footnotetext{
1 Universidade Federal do Amazonas (UFAM). Coari - AM. *E-mail: lucas.enf.almeida@hotmail.com

2 Universidade do Norte (UNINORTE), Manaus - AM.

${ }^{3}$ Secretaria Municipal de Saúde de Boa Vista dos Ramos - AM.

${ }^{4}$ Secretaria Municipal de Saúde de Barreirinha - AM.
} 


\section{RESUMEN}

Objetivo: Determinar la prevalencia de la Hiperplasia Prostática Benigna y los principales síntomas del aparato genito urinario en individuos que hacen uso del servicio público de salud, residentes en el municipio de Coari, Amazonas. Métodos: Se trata de un estudio transversal, descriptivo y observacional realizados con 106 hombres a partir de 40 años. Resultados: La prevalencia de Hiperplasia Prostática Benigna en la población masculina de Coari - Amazonas fue de 51,9\%. Los hombres con un rango de edad de 61 a 70 años y la edad de 71 años o más tuvieron las mayores tasas de cambio de volumen de la próstata El escore de la intensidad de los síntomas en el tracto urinario inferior encontrado en la mayoría de los individuos investigados fueron leves y el grado de disfunción La eréctil fue de leve a moderado. Conclusión: La Hiperplasia Prostática Benigna fue predominante en la mayoría de los hombres mayores de 40 años que hicieron uso de los servicios públicos de salud del municipio de Coari y la intensidad de los síntomas del aparato genito-urinario fueron de leve a moderada. Estos datos sirven de alerta para los profesionales de salud del municipio sobre la presencia de ese agravio y muestran la necesidad de intensificar o continuar las actividades de salud dirigidas a este público.

Palabras clave: Hiperplasia prostática, Salud del hombre, Sistema urinario.

\section{INTRODUÇÃO}

Caracterizada pelo crescimento benigno das células prostáticas, a Hiperplasia Prostática Benigna (HPB) é uma das enfermidades mais comuns entre os homens, que acarreta no aumento do tamanho da próstata (FILHO RFF, et al., 2017). Segundo a literatura, estima-se que a prevalência de HPB em homens corresponda a $8 \%$ na terceira década de vida, tornando-se superior a $80 \%$ nos indivíduos com idade acima de 80 anos (BRASIL, 2018).

A incidência de Hiperplasia Prostática Benigna é crescente com o avançar da idade e devido ao aumento da expectativa de vida da população masculina, esta doença tem se destacado entre as morbidades que mais acometem o sexo masculino durante a velhice (PIMENTA RCA, et al., 2013; NUNES RLV, et al., 2017). Sua etiologia ainda não foi totalmente elucidada, porém, fatores não modificáveis como idade e genética, bem como, modificáveis como distúrbios hormonais e metabólicos, obesidade, sedentarismo e outros, podem influenciar o surgimento ou não da HPB (PATELE ND e PARSONS JK, 2014).

As manifestações clínicas da Hiperplasia Prostática Benigna estão associadas principalmente aos sintomas do trato urinário inferior (STUI), que podem ser causados pelo estreitamento do canal uretral decorrente da alteração do volume da próstata ou pela ampliação do tônus da musculatura lisa da uretra (SILVA RN, et al., 2014; FILHO RFF, et al., 2017).

Os STUI são categorizados em três grupos: 1) Armazenamento: polaciúria, incontinência urinária enurese noturna e noctúria. 2) Esvaziamento: hesitação, jato fraco, bífido ou intermitente, gotejamento terminal e esforço miccional. 3) Pós-miccionais: gotejamento pós-miccional e tenesmo vesical (BRASIL, 2015). Dependendo da intensidade dos sintomas estes podem impactar negativamente na qualidade de vida do homem (SILVA RN, et al., 2014).

Sobre o diagnóstico da Hiperplasia Prostática Benigna, existem diversas opções, sendo geralmente clínico ao levantar informações sobre o histórico da doença, exame físico, aplicação do Escore Internacional de Sintomas Prostáticos (I-PSS: International Prostate Symptom Score) e Índice Internacional de Função Erétil (IIFE: International Index of Erectile Function), aliado a exames complementares como exame retal digital, exame de urina, de antígenos prostáticos específicos, imagens e outros (KIM EH, et al., 2016).

Em relação ao tratamento, dependendo do grau dos sintomas e obstrução vesical, este pode ser farmacológico, por exemplo, com medicamentos bloqueadores alfa-adrenérgicos que estimulam 0 relaxamento da musculatura da uretra e inibidores da 5-alfa redutase que ocasionam a diminuição do volume prostático ou pode-se recorrer ao procedimento cirúrgico nos casos mais graves, sendo este utilizado como último recurso (LANGAN RC, 2019). 
Desse modo, o diagnóstico e o tratamento precoce para HPB são necessários para o restabelecimento da saúde do homem, onde as políticas de saúde do Brasil voltada à população masculina possuem sua relevância ao garantir assistência integral a este grupo pelo sistema único de saúde (SEPARAVICH MA e CANESQUI AM, 2013)

Após o exposto, este estudo justifica-se pela relevância da temática e a escassez de estudos nas bases de dados científicas, sobre a HPB no contexto das populações Amazônicas. Dessa forma, faz-se necessário conhecer mais sobre a prevalência dessa doença nessas populações, bem como suas repercussões clínicas e sociais. Com isso, o objetivo do estudo foi determinar a prevalência da Hiperplasia Prostática Benigna e os principais sintomas do aparelho genito-urinário em indivíduos que fazem uso do serviço público de saúde, residentes no município de Coari, Amazonas.

\section{MÉTODOS}

Trata-se de um estudo transversal, descritivo e observacional, realizado com a população do sexo masculino do município de Coari - Amazonas, que era atendida na Policlínica Dr. Roque Juan Dell Oso e nas 12 (doze) Unidades Básicas de Saúde (UBSs) da respectiva cidade, sendo uma delas destinada ao público ribeirinho). O estudo foi submetido ao julgamento do Comitê de Ética em Pesquisa da Universidade Federal do Amazonas e aprovado conforme CAAE: 84449718.8.0000.5020.

Previamente, os indivíduos foram informados sobre a pesquisa e após o esclarecimento da importância do estudo, foi solicitado a permissão e consentimento, através da assinatura do Termo de Consentimento Livre e Esclarecido (TCLE), conforme rege a Resolução 466/12 do Conselho Nacional de Saúde (CNS). Este estudo compreendeu uma amostra de 250 homens usuários do serviço público de saúde que se encaixaram nos critérios de inclusão e exclusão.

Os critérios de inclusão foram homens, com idade igual ou superior a 40 anos de idade e cadastrados no Sistema Único de Saúde, com capacidade cognitiva preservada, aptos para responder o questionário e, que aceitaram participar através da assinatura do TCLE.

Os critérios de exclusão foram os que não participaram das etapas de coleta de informações e seguimento ambulatorial para realização da entrevista e exames; os que passaram por cirurgia urológica previa, neste caso prostatectomia; portadores de doenças crônico-degenerativas, comprometendo o Sistema Urogenital ou a cognição; aqueles que não residiam no município de Coari; os que estavam utilizando medicações específicas para patologias crônicas, com alteração do aparelho urogenital, como diuréticos e outros.

Objetivando uniformizar a avaliação clínica do paciente portador ou não de sintomas relacionados à HBP, foram utilizados questionários semiestruturados, compostos pelo I-PSS (BERGER M, et al., 1999) e o Índice Internacional de Função Erétil (IIFE: International Index of Erectile Function) (ROSEN RC, et al., 1997). O IPSS é composto por 7 questões com escores que, quando somados, refletem a intensidade dos STUI no último mês (escores de 0 a 7 indicam sintomas levas, 8 a 19 moderados, e 20 a 35 graves), além de uma oitava questão que avalia a qualidade de vida relacionados aos sintomas (escores variam entre 0-6 e indicam desde "ótimo" até "péssimo") (BERGER M, et al., 1999).

O IIFE é composto por 6 questões, e cada uma é pontuada de 0 a 5 de acordo com a resposta do entrevistado. $\mathrm{O}$ grau de disfunção erétil é atribuído conforme as somas das respostas, podendo ser de 26 a 30, considerado função erétil normal, 22 a 25 leve, 17 a 21 leve a moderada, 11 a 16 moderada e 1 a 10 grave (ROSEN RC, et al., 1997).

O questionário foi empregado pelo entrevistador da pesquisa, e respondido pelo entrevistado, obtendo dados sobre a identificação deste, as condições socioeconômicas em que vivem, além da sintomatologia de distúrbio geniturinários apresentada. Após entrevista do paciente estudado, o mesmo foi encaminhado para realizar um exame ecográfico de próstata (EEP) via abdominal (VA) ou retal (VR). A via retal foi a preferida em casos de pacientes que estavam apresentando sintomas obstrutivos importantes, pacientes negros, obesos ou com dificuldade para retenção de urina para avaliação via abdominal. Também foi via de preferência para os pacientes acimas de 60 anos. 
Os exames foram realizados gratuitamente na Policlínica Dr. Roque Juan Dell Oso e na Clínica de Medicina Ocupacional de Coari - CEMOC em convênio, onde era uma parceira da pesquisa. Para determinar a prevalência de hiperplasia foi considerado o volume prostático aumentado (VPA), o peso calculado pelo aparelho ultrassonográfico $>30$ gramas e volume prostático normal $(V P N)<30$ gramas. O peso foi calculado pela ultrassonografia, capturando a imagem da glândula, realizando as três medidas (altura, largura e comprimento), onde foi dado o volume total e multiplicado pela constante 1,05 . Os aparelhos de ultrassonografias utilizados foram da Marca Medison, Sonoace com transutores Convexos e Endocavitários.

Para análise estatística os dados foram descritos por frequência absoluta e relativa. Ademais foram empregados os testes estatísticos adequados para detectar ou não as diferenças entre as proporções amostrais entre o grupo objeto de estudo. O programa utilizado para as análises foi o software estatístico Statistical Package for Social Sciences 20 - SPSS. A significância estatística foi aceita ao nível de 95\%.

\section{RESULTADOS}

Participaram do estudo 106 homens, onde 102 (96,2\%) residiam em zona urbana e apenas $04(3,8 \%)$ na área rural do município de Coari - Amazonas. Estes tinham idade entre 40 anos a 85 anos, com média de idade de 59,42 anos. A prevalência de hiperplasia prostática benigna na população masculina de Coari Amazonas foi de $51,9 \%$ entre os participantes do estudo (Gráfico 1 ).

Gráfico 1 - Prevalência de Hiperplasia Prostática Benigna na população masculina. Coari - Amazonas, 2018.

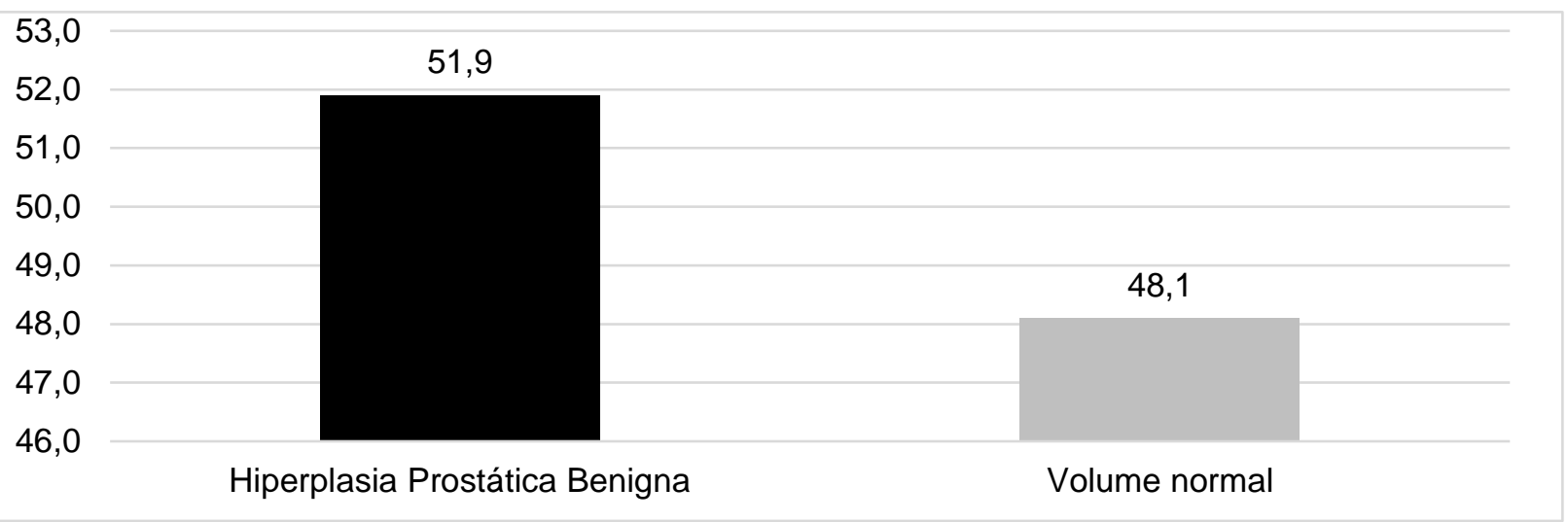

Fonte: Almeida LS, et al., 2018.

Dentre os 55 casos de HPB encontrados, a média de idade foi de 64,49 anos e o peso médio da próstata foi de $38,17 \mathrm{~g}$ por via transretal. De acordo com a Tabela 1, as alterações do volume da próstata foram encontradas em $26,1 \%$ dos participantes que tinham idade entre 40 a 50 anos, em $40,0 \%$ entre os que tinham 51 a 60 anos, em $66,7 \%$ naqueles que com 61 a 70 anos e em $93,8 \%$ nos que tinham idade igual ou superior a 71 anos, sendo as duas últimas categorias com as maiores taxas de alteração.

Tabela 1 - Volume prostático da população masculina, segundo idade. Coari - Amazonas, 2018.

\begin{tabular}{cccc}
\hline Faixa etária & Volume da Próstata & $\mathbf{n}$ & $\%$ \\
\hline \multirow{2}{*}{$40-50$ anos } & Aumentado & 06 & 26,1 \\
& Normal & 17 & 73,9 \\
$51-60$ anos & Aumentado & 16 & 40,0 \\
& Normal & 24 & 60,0 \\
$61-70$ anos & Aumentado & 18 & 66,7 \\
& Normal & 09 & 33,3 \\
$\geq 71$ anos & Aumentado & 15 & 93,8 \\
& Normal & 01 & 6,3 \\
\hline
\end{tabular}

Fonte: Almeida LS, et al., 2018. 
Os sintomas urinários de armazenamento, esvaziamento e pós-miccional mais frequentes entre os participantes com e sem Hiperplasia Prostática Benigna foram noctúria $(57,5 \%)$, jato fraco $(29,2 \%)$ e tenesmo vesical $(29,2 \%)$. Os menos citados foram incontinência urinária $(17,0 \%)$, hesitação $(12,3)$ e gotejamento pósmiccional (11,3\%) (Tabela 2).

Tabela 2 - Distribuição dos sintomas urinários de armazenamento, esvaziamento e pós-miccional na população masculina. Coari - Amazonas, 2018.

\begin{tabular}{ccccc}
\hline \multirow{2}{*}{ Sintomas } & \multicolumn{2}{c}{ Sim } & \multicolumn{3}{c}{ Não } \\
\cline { 2 - 5 } & $\mathbf{n}$ & $\%$ & $\mathbf{n}$ & $\%$ \\
\hline Armazenamento & & & & \\
\hline Polaciúria & 27 & 25,5 & 79 & 74,5 \\
Noctúria & 61 & 57,5 & 45 & 42,5 \\
Incontinência Urinária & 18 & 17,0 & 88 & 83,0 \\
\hline Esvaziamento & & & & \\
\hline Jato Fraco & 31 & 29,2 & 75 & 70,8 \\
Hesitação & 13 & 12,3 & 93 & 87,7 \\
Esforço Miccional & 19 & 17,9 & 87 & 82,1 \\
Gotejamento Terminal & 16 & 15,1 & 90 & 84,9 \\
Jato Bífido & 17 & 16,0 & 89 & 84,0 \\
Disúria & 21 & 19,8 & 85 & 80,2 \\
\hline Pós-Miccional & & & & \\
\hline Tenesmo Vesical & 31 & 29,2 & 75 & 70,8 \\
Gotejamento Pós-miccional & 12 & 11,3 & 94 & 88,7 \\
\hline
\end{tabular}

Fonte: Almeida LS, et al., 2018.

A Tabela 3 revelou que segundo o Escore Internacional de Sintomas Prostáticos a intensidade dos sintomas mais frequentes entre os participantes com e sem HPB foram os leves $(68,9 \%)$, seguidos do moderado $(23,6 \%)$ e grave $(7,5 \%)$. Em relação ao Índice Internacional de Função Erétil evidenciou-se a disfunção leve a moderado (34,0\%), normal (25,5\%) e disfunção moderada (20,8\%).

Tabela 3 - Classificação dos sintomas do aparelho genito-urinário na população masculina de Coari Amazonas.

\begin{tabular}{lcc}
\hline \multicolumn{1}{c}{ Classificação } & n & $\%$ \\
\hline Escore Internacional de Sintomas Prostáticos & & \\
\hline Sintomas Leves & 73 & 68,9 \\
Sintomas Moderado & 25 & 23,6 \\
Sintomas Grave & 8 & 7,5 \\
\hline Índice Internacional de Função Erétil & & \\
\hline Normal & 27 & 25,5 \\
Disfunção Leve & 17 & 16,0 \\
Disfunção Leve a Moderado & 36 & 34,0 \\
Disfunção Moderado & 22 & 20,8 \\
Disfunção Grave & 04 & 3,8 \\
\hline
\end{tabular}

Fonte: Almeida LS, et al., 2018.

Quando se relacionou o grau de disfunção erétil com o peso prostático acima de $30 \mathrm{~g}$, notou-se que $38,2 \%$ dos participantes apresentaram sintomas leve a moderado, seguidos dos sintomas só moderados $(23,6)$, leves (16,4\%) e normal (16,4\%) (Gráfico 4). 
Gráfico 4 - Relação do grau de disfunção erétil e peso prostático $>30 \mathrm{~g}$ da população masculina de Coari - Amazonas.

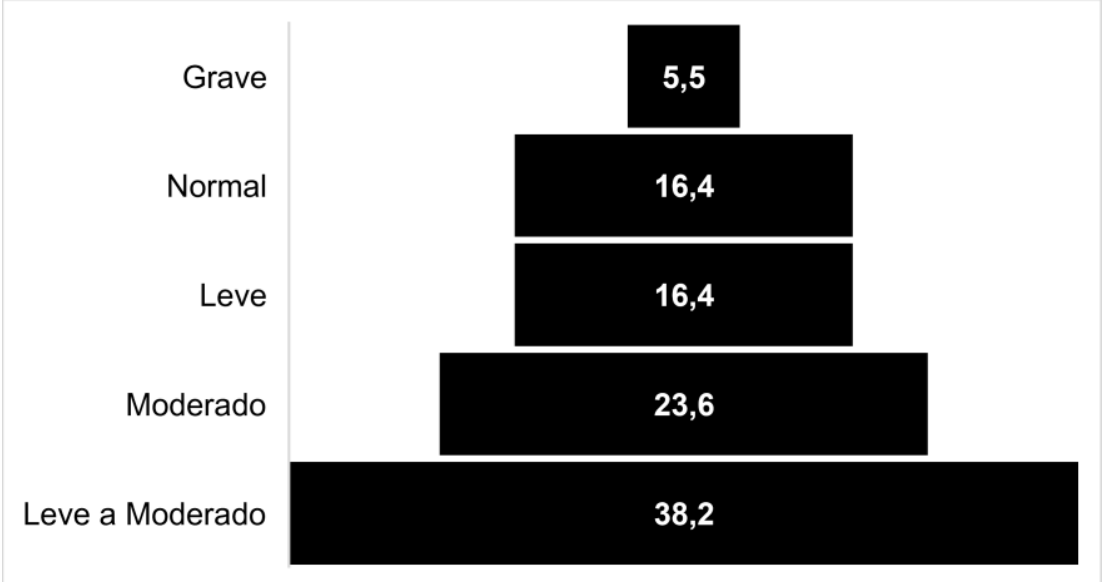

Fonte: Almeida LS, et al., 2018.

Ao aplicar o teste de Pearson entre as variáveis peso prostático e escore I-PSS de sintomas prostáticos, identificou-se que não houve uma associação estatística $(0,463)$. Quando se correlacionou as variáveis idade e peso prostático, também não houve uma associação estatística $(0,469)$ (Tabela 5).

Tabela 5 - Correlação entre peso prostático, escore I-PSS de sintomas prostático e idade da população masculina de Coari - Amazonas.

\begin{tabular}{cc} 
Correlações & Valor de $\mathbf{p}$ \\
\hline Peso Prostático em gramas X Sintomas Urinários - IPSS & 0,463 \\
Peso Prostático em gramas X Idade & 0,469
\end{tabular}

Fonte: Almeida LS, et al., 2018.

\section{DISCUSSÃO}

Na região norte do Brasil, mais especificamente no estado do Amazonas, o rastreamento da Hiperplasia Prostática Benigna ainda tem sido pouco explorado pelos pesquisadores. No entanto, através desta pesquisa foi possível obter um bom panorama dessa doença e os principais sintomas gerados no aparelho genitourinário da população masculina residente na cidade de Coari.

No presente estudo a hiperplasia prostática benigna foi prevalente na maioria dos homens que foram pesquisados $(51,9 \%)$. Esses dados confirmam a incidência dessa enfermidade entre os homens e alertam os profissionais de saúde do município sobre a presença desse agravo no público atendido (FILHO RFF, et al., 2017).

A média de idade 64,49 anos e o peso médio da próstata de 38,17gramas entre os 55 pacientes com HPB foi similar ao encontrado em outros estudos. Pesquisas como de Júnior AMC, et al. (2015), Byun HK, et al. (2012) e Schenk JM, et al. (2013), apontam para uma idade média de 59,5 anos a 62,5 anos. Da mesma maneira, o peso médio da próstata tem variado entre 42,9 gramas a 52 gramas (JÚNIOR AMC, et al., 2015; BELLUCCI CHS, 2005; SANTOS VCT, et al., 2006).

As taxas de alterações do volume da próstata foram crescentes com o avançar da idade $(26,1 \% ; 40,0 \%$; $66,7 \% ; 93,8 \%)$, sendo elevados entre os participantes que tinham a faixa etária entre 61 a 70 anos (66,7\%) e igual ou superior a 71 anos (93,8\%). Esses dados foram semelhantes ao identificado na pesquisa de Silva IC e Silva LB (2016), onde ao realizar o levantamento da frequência de internações na região centrooeste do Brasil, notou-se que as hospitalizações por HPB aumentavam com o envelhecimento (de $0,06 \%$ para $34,54 \%$ ), ocorrendo assim, os casos com maior constância entre os pacientes com idade entre 60 a 69 anos $(39,62 \%)$ e 70 a 79 anos $(34,54 \%)$. 
O achado também foi similar a um estudo internacional realizado na China, no qual a prevalência de Hiperplasia Prostática Benigna elevou-se com a idade, aumentando de 2,17\% em homens com faixa etária de 40 a 44 anos para $31,11 \%$ em homens de 80 anos ou mais (YUE L, et al., 2019).

Desta forma, a evidência da faixa etária do estudo corrobora ao encontrado na literatura, que segundo a mesma, o envelhecimento do organismo humano associado ao aumento da expectativa de vida da população masculina tem influenciado na incidência dessa morbidade no sexo masculino e isto pode estar relacionado ao processo de transição epidemiológica e aos avanços científicos e tecnológicos na área da saúde (FILHO RFF, et al., 2017; PIMENTA RCA, et al., 2013; CAMARGOS MCS e GONZAGA MR, 2015).

Quando se analisou as principais manifestações clínicas no trato urinário inferior no estudo, evidenciou-se que os sintomas mais frequentes foram os de armazenamento (noctúria 57,5\%). Este resultado sustenta o apontado na pesquisa belga que investigou os sintomas do trato urinário inferior em homens com idade maior ou igual a 40 anos, em que a noctúria foi o sintoma mais citado pelos participantes $(69,2 \%)$ (RIDDER D, et al., 2015).

A noctúria é uma das sintomatologias mais frequentes em homens que sofrem de STUI. A necessidade de levantar-se durante a noite para esvaziar a bexiga duas ou mais vezes, acaba comprometendo a qualidade do sono do paciente e isto consequentemente, impacta de forma negativa na qualidade de vida do indivíduo, podendo interferi em suas atividades diárias (MIRANDA EP, et al., 2014).

Ao avaliar o grau de comprometimento funcional prostático através dos relatos registrados no Escore Internacional de Sintomas Prostáticos, a maioria dos homens investigados $(68,9 \%)$ apresentaram sintomas leves. Um estudo realizado com 155 homens portadores de Hiperplasia Prostática Benigna da região Sul do Brasil, apresentou predominância de sintomas moderados, bem como, outro estudo de 2015 da região nordeste indicou a maioria dos sintomas também moderados sendo dissonantes dos resultados encontrados nesta atual investigação com paciente da região norte do país (BELLUCCI CHS, 2005; JÚNIOR AMC, et al., 2015).

A predominância dos sintomas moderados nos estudos mencionados sugeriu que os homens destas pesquisas eram diagnosticados quando a doença já está avançada, o que para Bellucci CHS (2005) dificulta a abordagem terapêutica contra a doença. Entretanto, nesta pesquisa, a prevalência de sintomas leves sugere que o diagnóstico entre homens de Coari tenha sido precoce, facilitando não apenas o tratamento, mas minimizou os gastos com a saúde, as chances de danos irreversíveis, assim como, o aumento da qualidade de vida.

Ao analisar o Índice Internacional de Função Erétil, prevaleceu no estudo o grau de disfunção erétil de leve a moderado com $34,0 \%$. A interferência na atividade sexual é um fato que também compromete a qualidade de vida do homem, onde os STUI decorrentes as HPB podem estar relacionados a essa condição, onde a investigação realizada com os pacientes da Polônia com HPB confirmou uma forte correlação entre o aumento dos sintomas do I-PSS com as manifestações clinicas mais profundos de disfunção erétil (DUTKIEWICZ S, et al., 2012). Diante dessa evidência, os sintomas leves a moderados de disfunção erétil identificados na pesquisa podem estar relacionados a baixa presença de sintomas graves de STUI nos entrevistados.

Nesta pesquisa nem o escore I-PSS de sintomas prostáticos, nem a idade tiveram relação estatística significativa forte quando correlacionadas com o peso da próstata em gramas. Em um estudo realizado na Suécia por Vesely S, et al. (2003), concluiu-se que não há correlação significativa forte entre escore I-PSS e peso prostático ou escore I-PSS e idade, corroborando assim com os resultados encontrados.

Outros trabalhos ainda, vem reforçar os resultados nesta investigação, uma vez que também não encontraram correlação significativa forte entre escore I-PSS, idade e peso prostático (UDER EL, et al., 2012; BOTTORFF JL, et al., 2015).

Os valores nesta pesquisa podem ser ainda maiores, levando em consideração, que apenas uma parcela dessa população buscou os serviços de saúde durante a execução do estudo. Moreira RLSF et al. (2014) afirma que o sexo masculino é um grupo que possui resistência para buscar os serviços de saúde, recorrendo ao mesmo apenas quando os sintomas se agravam. Apesar da criação da Política Nacional de Atenção 
Integral à Saúde do Homem está ainda não tem sido muito efetiva, pois vários são os fatores que interferem na assistência à saúde do homem, tais como: a ausência do homem na atenção primária, déficit de autocuidado com a saúde, medo, incompatibilidade de horário, dificuldade de acesso aos serviços de saúde e outros (MOREIRA RLSF, et al., 2014; CAVALCANTI JRD, et al., 2014).

Desta maneira, muitos são os desafios para o reconhecimento precoce dos agravos que acometem os homens não sendo diferente no município de Coari, principalmente no que diz respeito a rastreio da Hiperplasia Prostática Benigna na população. Ainda que a HPB não seja uma doença com alta letalidade, a sua morbidade e riscos de complicação preocupam, pois, podem ocasionar na redução da qualidade de vida dos acometidos, além de causar aumento dos gastos públicos com a saúde.

Neste sentindo, recomenda-se que os profissionais de saúde incentivem um estilo de vida saudável aos seus pacientes, mesmo nos assintomáticos, porque essas atitudes podem diminuir a propensão desses indivíduos desenvolverem HPB ou qualquer outra doença, principalmente em uma época crescente da longevidade masculina e incidência de doenças crônicas (YOO TK, et al., 2012; PARSONS JK, 2010).

Vale acrescentar, que as limitações encontradas no estudo, estão relacionadas principalmente a discussão dos resultados evidenciados, onde recorreu-se em maior parte a literatura internacional devido ao número reduzido de pesquisas transversais com esta temática em âmbito nacional. Todavia, espera-se que essa investigação possa contribuir para a comunidade cientifica ao desmitificar a ocorrência da Hiperplasia Prostática Benigna na população masculina pertencente a um município da região norte do Brasil e incentivar pesquisas que possam rastrear a HPB em todo território brasileiro.

\section{CONCLUSÃO}

A Hiperplasia Prostática Benigna foi prevalente na maioria dos homens acima de 40 anos que fizeram uso dos serviços públicos de saúde do município de Coari. As taxas de alterações do volume da próstata foram crescentes com o avançar da idade, com predominância entre os participantes que tinham a faixa etária entre 61 a 70 anos e igual ou superior a 71 anos.

Noctúria foi o sintoma mais citado pelos indivíduos pesquisados e a intensidade dos sintomas do aparelho genito-urinário foram de leve a moderado (I-PSS: leve; IIFE: leve a moderado). Portanto, esses dados servem de alerta para os profissionais de saúde do município sobre a presença desse agravo entre os moradores locais e mostram a necessidade de intensificar ou continuar as atividades de promoção, prevenção e recuperação da saúde voltadas a este público, haja vista, que o diagnóstico precoce evita custos desnecessários, otimiza o tratamento e minimiza as consequências advindas dessa doença que podem comprometer a qualidade de vida do paciente coariense.

\section{REFERÊNCIAS}

1. BELLUCCI CHS. Associação entre sintomas e achados ultrassonográficos na hiperplasia prostática benigna [trabalho de conclusão de curso]. Florianópolis: Universidade Federal de Santa Catarina; 2005.

2. BERGER M, et al. Validação estatística do escore internacional de sintomas prostáticos (I-PSS) na língua portuguesa. J Bras Urol. 1999; 25(2): 225-234.

3. BOTTORFF JL, et al. An Updated review of interventions that inclube promotions of physical activity for adult men. Sport Med. 2015; 45(6): 775-800.

4. BRASIL. Hiperplasia Prostática Benigna. 2015. In: RegulaSUS. Brasília: Ministério da Saúde. Disponível em: https://www.ufrgs.br/telessauders/documentos/protocolos_resumos/urologia_resumo_hiperplasia_prostatica_benign a_TSRS.pdf. Acesso em:25 de abr. 2020.

5. BRASIL. Hiperplasia prostática benigna. 2018. In: Manual MSD. Brasília: Ministério da Saúde. Disponível em: https://www.msdmanuals.com/pt/profissional/dist\%C3\%BArbios-geniturin\%C3\%A1rios/doen\%C3\%A7aprost\%C3\%A1tica-benigna/hiperplasia-prost\%C3\%A1tica-benigna-hpb. Acesso em: 23 de abr. 2020.

6. BYUN HK, et al. Relationships between prostate-specific antigen, prostate volume, and components of metabolic syndrome in healthy Korean men. Korean J Urol. 2012; 53(4): 471-477.

7. CAMARGOS MCS, GONZAGA MR. Viver mais e melhor? Estimativas de expectativa de vida saudável para a população brasileira. Cad. Saúde Pública. 2015; 31(7): 1460-1472.

8. CAVALCANTI JRD, et al. Assistência Integral a Saúde do Homem: necessidades, obstáculos e estratégias de enfrentamento. Esc Anna Nery 2014; 18(4): 628-634. 
9. DUTKIEWICZ S, et al. Assessing the Influence of Benign Prostatic Hyperplasia (BPH) on Erectile Dysfunction (ED) among patients in Poland. Cent European J Urol. 2012; 65(3): 135-138.

10. FILHO RFF, et al. Hiperplasia prostática benigna: revisão de literatura. R. Interd. 2017; 10(1): $200-204$.

11. JÚNIOR AMC, et al. Correlação entre idade, intensidade de sintomas prostáticos e achados ultrassonográficos. Rev Bras Promoç Saúde. 2015; 28(1): 44-49.

12. KIM EH, et al. Management of Benign Prostatic Hyperplasia. Annual Review of Medicine. 2016; 67(1): $137-151$.

13. LANGAN RC. Benign Prostatic Hyperplasia. Prim Care. 2019; 46(2): 223-232.

14. MIRANDA EP, et al. Nocturia is the lower urinary tract symptom with greatest impact on quality of life of men from a community setting. Int Neurourol J. 2014; 18(2): 86-90.

15. MOREIRA RLSF, et al. Dificuldades de inserção do homem na atenção básica a saúde: a fala dos enfermeiros. Esc. Anna Nery. 2014; 18(4): 615-621.

16. NUNES RLV, et al. Contemporary surgical treatment of benign prostatic hyperplasia. Rev Assoc Med Bras. 2017; 63(8): 711-716.

17. PARSONS JK. Benign Prostatic Hyperplasia and Male Lower Urinary Tract Symptoms: Epidemiology and Risk Factors. Current Bladder Dysfunction Reports. 2010; 5(4): 212-218.

18. PATELE ND, PARSONS JK. Epidemiology and etiology of benign prostatic hyperplasia and bladder outlet obstruction. Indian J Urol. 2014; 30(2): 170-176.

19. PIMENTA RCA, et al. Rastreamento da hiperplasia prostática benigna. Ciência ET Praxis. 2013; 6(12): 35-38.

20. RIDDER D, et al. Urgency and other lower urinary tract symptoms in men aged $\geq 40$ years: a Belgian epidemiological survey using the ICIQ-MLUTS questionnaire. Int J Clin Pract. 2015; 69 (3): 358-365.

21. ROSEN RC, et al. The international index of erectile function (IIEF): a multidimensional scale for assessment of erectile dysfunction. Urology. 1997; 49(6): 822-830.

22. SANTOS VCT, et al. Current role of transrectal ultrasonography in the early detction of prostate câncer. Radiol Bras. 2006; 39(3): 182-92.

23. SCHENK JM, et al. Should modest elevations in prostate-specific antigen, international prostate symptom score, or their rates of increase over time be used as surrogate measures of incident bening prostatic hyperplasia? Am $\mathrm{J}$ Epidemiol. 2013;178(5): 741-751.

24. SEPARAVICH MA, CANESQUI AM. Saúde do homem e masculinidades na Política Nacional de Atenção Integral à Saúde do Homem: uma revisão bibliográfica. Saúde Soc. 2013; 22(2): 415-428.

25. SILVA IC, SILVA LB. Frequência de internação por HPB (hiperplasia prostática benigna) na região centro-oeste do Brasil. ICESP. 2016; 1(1) 933-936.

26. SILVA RN, et al. Ultrassonografia no diagnóstico e na avaliação da hiperplasia prostática benigna. RBUS. 2014; 16(01): 46-49.

27. UDER El, et al. The relationship between prostate volume and international prostate symptom score in Africans with bening prostatic hyperplasia. Niger J Med. 2012; 21(3): 290-295.

28. VESELY S, et al. Relationship between age, prostate volume, prostate specific antigen, symptom score and uroflowmetry in men with lower urinary track symptoms. Scand J Urol Nephrol. 2003;37(4):322-8.

29. YOO TK, et al. Benign prostatic hyperplasia: from bench to clinic. Coreano J Urol. 2012; 53(3): 139-148.

30. YUE L, et al. Prevalence and heritability of benign prostatic hyperplasia and LUTS in men aged 40 years or older in Zhengzhou rural areas. Prostate. 2019; 79(3): 312-319. 